\title{
MODIFICATION AND TESTING OF AN ENGINE AND FUEL CONTROL SYSTEM FOR A HYDROGEN FUELLED GAS TURBINE
}

\author{
H. H.-W. Funke ${ }^{1}$, S. Börner ${ }^{1}$, P. Hendrick ${ }^{2}$, and E. Recker ${ }^{3}$ \\ ${ }^{1}$ Department of Aerospace Technology \\ Aachen University of Applied Sciences \\ Hohenstaufenallee 6, Aachen 52064, Germany \\ ${ }^{2}$ Université Libre de Bruxelles \\ Av. F. D. Roosevelt 50, Brussels 1050, Belgium \\ ${ }^{3}$ Royal Military School of Belgium \\ Av. de la Renaissance 30, Brussels 1000, Belgium
}

\begin{abstract}
The control of pollutant emissions has become more and more important by the development of new gas turbines. The use of hydrogen produced by renewable energy sources could be an alternative. Besides the reduction of NOx emissions emerged during the combustion process, another major question is how a hydrogen fuelled gas turbine including the metering unit can be controlled and operated. This paper presents a first insight in modifications on an Auxiliary Power Unit (APU) GTCP 36300 for using gaseous hydrogen as a gas turbine fuel. For safe operation with hydrogen, the metering of hydrogen has to be fast, precise, and secure. So, the quality of the metering unit's control loop has an important influence on this topic. The paper documents the empiric determination of the proportional integral derivative (PID) control parameters for the metering unit.
\end{abstract}

\section{INTRODUCTION}

Gas turbines as reliable, efficient, long-life, and light-weight propulsion systems will be parts and parcels of future aircraft development. Due to the narrowness of fossil fuels and international efforts on reducing the environmental pollution, there is inherent need for alternative renewable fuels. A possible alternative is (besides synthetic fuels) the application of hydrogen, if it is produced by using renewable energy sources (e.g., sun and wind energy).

This is an Open Access article distributed under the terms of the Creative Commons Attribution-Noncommercial License 3.0, which permits unrestricted use, distribution, and reproduction in any noncommercial medium, provided the original work is properly cited. 


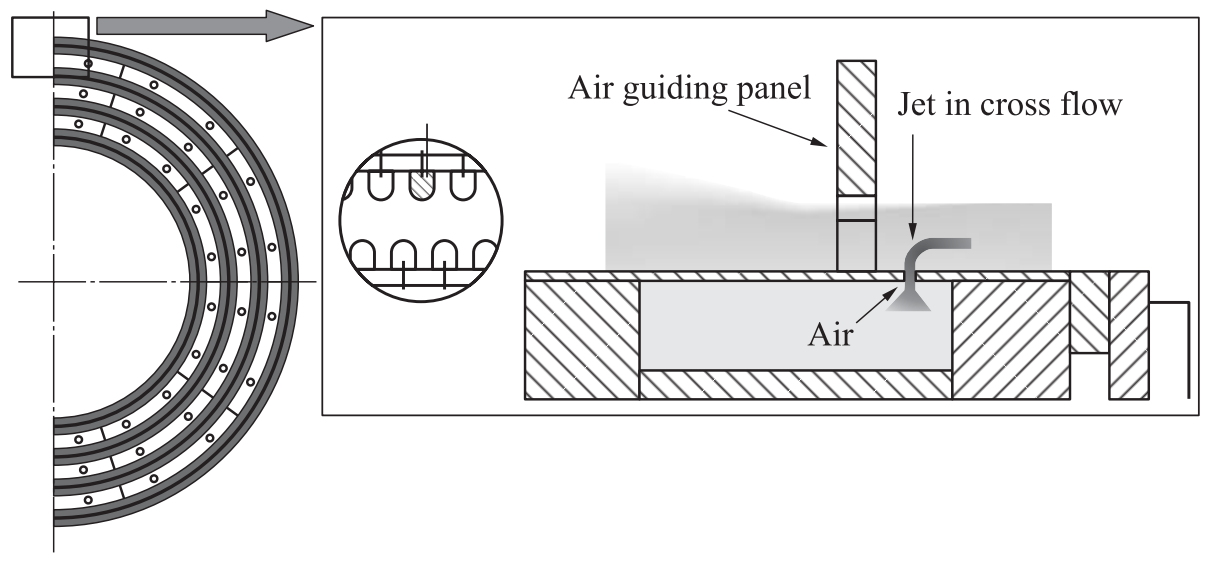

Figure 1 Micromix burning principle

By the use of hydrogen as a fuel for gas turbine engines, there are two major questions which have to be answered before the adaptation in aircraft industry:

1. How can hydrogen be burned efficient and stable under variable operation conditions with minimum possible NOx emissions while the safety risk of flashback is eliminated?

2. Which modifications for the control system and the fuel metering system have to be realized in order to allow the rapid and precise changes of engine power level as necessitated by aircraft operation conditions?

Since years, Aachen University of Applied Sciences (ACUAS) is working to solve these two major questions $[1,2]$. In order to achieve a substantial reduction of NOx emissions during the combustion process, the micromix burning principle has been developed. This principle for gaseous hydrogen [3-5] is based on the fluid mechanic phenomenon of combustion of jets in cross flow.

In the presented case of the micromix burning principle, the hydrogen is injected vertical into the airflow. The hydrogen burns in multiple miniaturized flames directly after the interaction of jets in the cross flow. Currently, the hydrogen chamber with about 1.6 MW of thermal power counts nearly 1600 miniaturized flames (Fig. 1). Through this miniaturization of the flame length and flame temperature, the burning principle achieves a lowering of the reaction temperature and a reduction of the residence time of reactants in the flame region and, therefore, reduces the NOx formation [2]. Various experimental [4, 5] and numerical [6] investigations have been performed in order to understand the phenomena of the principle and their influence on the NOx formation. Due to the 
fact that this burning principle is designed as a nonpremixed concept, there is an inherent safety against flashback.

Besides optimizing the burning principle to lower NOx emissions, the control system and the metering unit of the gas turbine have to be modified and improved. The basic idea is to modify a kerosene-driven gas turbine into a hydrogen fuelled gas turbine. The operability of the hydrogen fuelled gas turbine should be similar to the kerosene fuelled turbine as much as possible. With the transition from kerosene to hydrogen, also a transition from liquid to gaseous fuel occurs. Due to this fact and strict safety requirements for hydrogen applications, the control and metering systems have to be also modified. One basic requirement to the control system is the fast and precise metering of hydrogen for safe ignition and for different operating conditions of gas turbines. In addition, the control loop has also to consider the mechanic and hydraulic behavior of the metering system.

With the implementation of the modified and improved control and metering system, the PID parameters of the control loop have to be set in order to guarantee the designated and secure operating/starting sequence of hydrogen engine. For testing the adjustments, first, the ignition tests have been performed and presented in this paper.

\section{ENGINE AND FUEL CONTROL MODIFICATION}

\subsection{Engine and Test Rig}

For the experimental work, two APU GTCP 36-300 are available at the university. The single-shaft gas turbine is used in different aircraft, such as AIRBUS A-320, and provides electrical and pneumatic power to the aircraft via a generator and an extra load compressor. The main components of the engine are the single-stage radial compressor, annular combustion chamber consisting of 6 single fuel nozzles, and a single-stage radial turbine. The engine is controlled by the Versa-

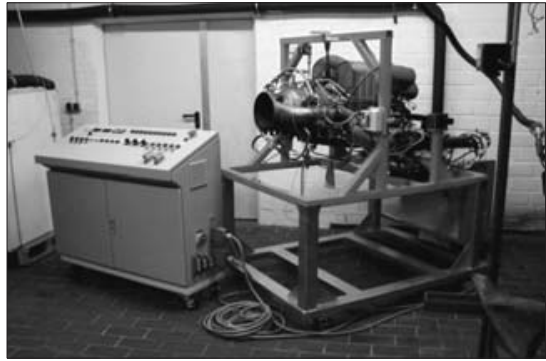

Figure 2 Auxiliary power unit GTCP $36-300$ in a mobile test rig tile Engine Control Box (VECB) built by DIEHL Aerospace GmbH. The APU can produce up to $335 \mathrm{~kW}$ during main engine start (MES) operation. To compare the influence of the changeovers for the hydrogen application on the gas turbine behavior and on the exhaust emissions, one APU has been modified whereas the other was still aimed for kerosene applications. To meet the require- 
ments of testing the gas turbine outside the laboratory for possible applications in industrial environment, the test rig was built as a mobile test rig (Fig. 2).

\subsection{Hydrogen Modifications}

To run the engine with hydrogen instead of kerosene, several modifications have been made. Since hydrogen cannot serve as a hydraulic medium for the loadcompressor guide vane actuator; therefore, an external hydraulic unit fulfilled these functions. The change of the combustion chamber was made in two steps. To get first impressions of the hydrogen-fuelled gas turbine behavior, the kerosene nozzles were replaced by gaseous ones. It is known from measurements that this modification will not have a significant impact on the NOx emissions compared to kerosene [2]. Later on, when the parameters governing NOx formation will be evaluated and the improved combustion-chamber design will be developed, a new combustion chamber based on the micromix principle will be installed in the engine. Also, the fuel metering system for gaseous hydrogen and the control software have been designed and improved based on the former control systems [7].

\subsection{Control Unit}

Figure 3 shows the optimized and hydraulically operated hydrogen metering unit. The hydrogen mass flow is controlled by the valve which is modified for hydrogen applications. This valve is traversed by a hydraulic cylinder whose position determines the hydrogen mass flow and is used as the process variable

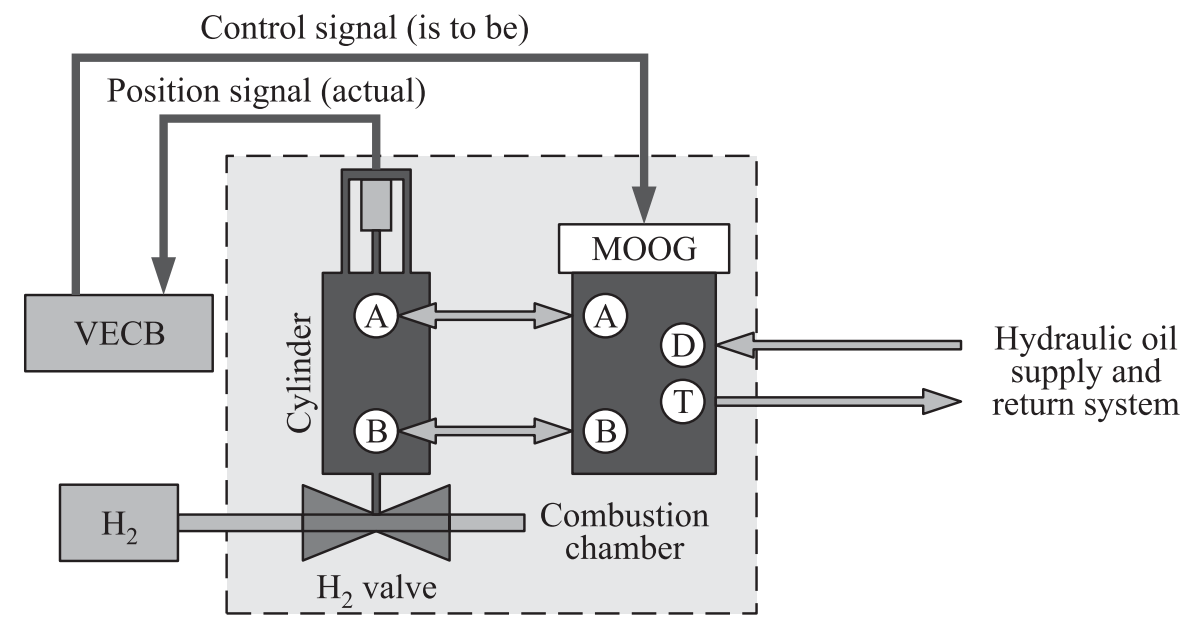

Figure 3 The hydrogen metering unit 
given back to the control unit. The cylinder operation is metered by a hydraulic MOOG valve. This valve constitutes the central part of the hydrogen metering unit and is actuated by the control unit.

Due to dynamic requirements to the hydrogen fuelled gas turbine, a very fast metering unit was designed and built. Besides the fuel metering valve, other valves important for safety reasons were also used. In the case of emergency or malfunction of the hydrogen gas turbine, the main fuel supply could be stopped automatically and manually. In addition, a fast thermocouple was implemented in the exhaust gas area for fast detection of ignition in the gas turbine.

\subsection{Control Software}

The test engine and the metering unit were controlled and operated by the VECB. The box was integrated in the mobile driver's control desk. The major function of the controller was to keep the engine speed constant at each load condition. The two essential operating conditions were:

(1) Environmental Control Supply (ECS): the APU delivers compressed air for the air condition; and

(2) MES: start of the main engine. Maximum power output of the APU.

The engine is controlled by different controllers. During the starting sequence, acceleration from $0 \%$ to $95 \%$ speed including engine ignition controlled by the first controller. Thereafter, the second closed-loop engine controller, which is referred to as on speed governor, takes over for all operating conditions of the APU. Its function is to keep the gas turbine speed constant in the range between $99 \%$ and $101 \%$ rotational speed, for each load condition by varying the fuel quantity, load-compressor guide vanes, and surge control valve. The function of the first controller during the acceleration process of the kerosene-fuelled gas turbine was to assume the required acceleration based on the reference acceleration map. The operation mode of the hydrogen-fuelled gas turbine during acceleration was different. The metering of hydrogen to the combustion chamber was controlled as a function of speed during the acceleration up to $95 \%$ rotational speed. Each speed step was attributed to a valve opening position and, therefore, the hydrogen mass flow. Between the two speed steps, the valve opening position was interpolated. An improvement point is that the integrated VECB controlled both the gas turbine and metering unit. Therefore, an additional PID (vlv_PID-Modul) controller has been implemented in the VECB and its software (Fig. 4). So, an additional and separate control loop for the metering unit could be avoided. The main control loop software of the gas turbine sent the requested opening position to the vlv_PID-Modul. The task of the PID-Modul was to assure the required hydrogen valve position by operating the MOOG valve. A position encoder measured the actual position of the valve 


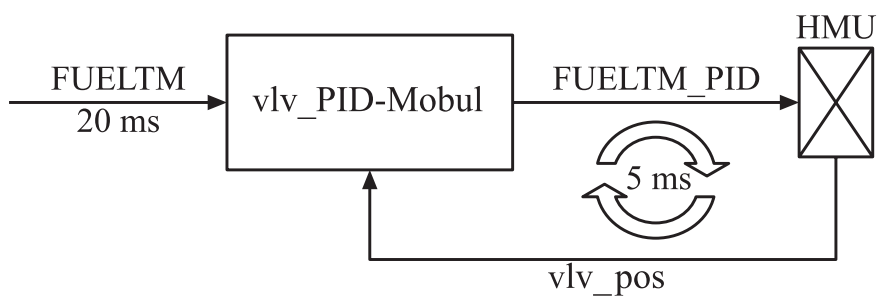

Figure 4 The control loop

(vlv_pos) and reported it to vlv_PID-Modul. If there was a spread between the actual and the required valve position, the vlv_ PID-Modul sent a control current (FUELTM_PID) to the MOOG valve in order to reach a minimization of the spread. The clocking of vlv_PID-Modul control loop was $5 \mathrm{~ms}$, whereas the main control loop software clocking was $20 \mathrm{~ms}$. Additionally, there were also few other safety changes in the engine control software for hydrogen applications. To make sure that there is no uncontrolled hydrogen in the combustion chamber, a Dry Crank has been implemented before the engine start. Therefore, the gas turbine was accelerated up to $5 \%$ rotational speed before the ignition and acceleration processes started. The ignition sequence has also been modified in the hydrogen controller version. The starting plug started directly at the beginning of gas turbine rotation. At 7\%, the hydrogen valve opened. To detect the ignition in a predefined window, the fast thermocouple mentioned above was used. In case of no detected ignition, the hydrogen supply was immediately stopped. For preventing the overheat of the combustion chamber resultant from a short temperature lowering, a time delay was implemented as well. In addition, the activation of safety valves in the hydrogen supply system was also implemented in the modified controller software.

\section{EMPIRICAL DETERMINATION OF THE PID CONTROL PARAMETERS FOR THE METERING UNIT}

\subsection{Requirements to the Metering Unit Control Loop}

With the effort to implement a hydrogen driven gas turbine with the same operating characteristics as a kerosene fuelled gas turbine, the main needs of the control loop were also defined. The main operating characteristics and requirements of the APU, especially during ignition and acceleration, are taken from the kerosene-fuelled operation (Fig. 5). Also, the FTM-value over time which is the unit of measurement for the requested kerosene mass flow is shown in Fig. 5. 


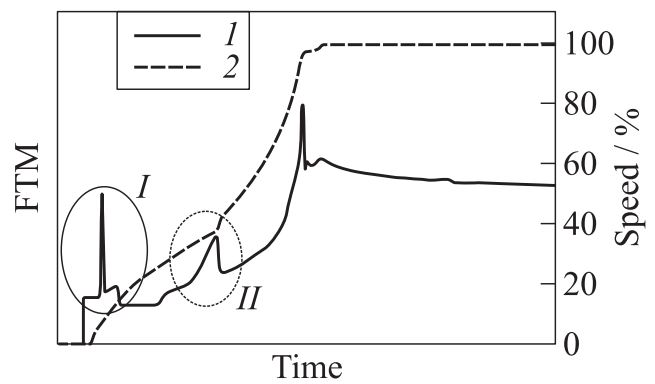

Figure 5 Kerosene reference run: $1-$ FTM, and $2-$ speed

The kerosene-fuelled gas turbine starts with a fuel spiking (border $I$ ) in order to ensure ignition. Due to the high reactivity of hydrogen and the risk of gas turbine overheat, this procedure can be eliminated in the controller software for hydrogen operation. In order to overpower the uncoupling of the starter and continuing acceleration, the control loop increases the FTM-value (border $I I$ ). For hydrogen implantation, these characteristics have to be observed with great accuracy: if the injected fuel mass flow is too high, the combustion chamber can be overheated. The ignition of hydrogen followed by the compensation of the dropped out starter presents the most critical phase during the starting sequence.

Due to the fact that the modifications are based on the main idea of feeding always the requested amount on energy for achieving the same gas turbine operation characteristics, the basic load of the metering unit and, therefore, the control unit is to guarantee:

$$
\dot{Q}_{\text {kerosene }}=\dot{Q}_{\text {hydrogen }} \Rightarrow \dot{m}_{\text {kerosene }} \Delta h u_{\text {kerosene }}=\dot{m}_{\text {hydrogen }} \Delta h u_{\text {hydrogen }} .
$$

As already mentioned, the position of the valve is the measure of the hydrogen mass flow. So, the control loop has to always assure the requested position of the valve for every engine operating condition. Also, the over- and undershooting of the valve has to be avoided, because this could result in overheating or a flame blow-off in the combustion chamber.

The transition from kerosene to hydrogen and from liquid to gaseous medium also includes the possibility of compressive behavior of hydrogen. This has to be foreseen by the design of the control loop characteristics. Also, the hydrogen reaction rate is higher than the kerosene reaction rate, in particular, at the ignition of the gas turbine. This has certainly an important influence. In order to get a first impression of the hydrogen-fuelled gas turbine operation and the corresponding requirements to the metering unit and control loop, first investigations were performed with a KHD T216 at ACUAS [7]. Figure 6 shows the difference between ignition of hydrogen and kerosene in the engine. Ignition of the hydrogen fuel has taken place after $0.02 \mathrm{~s}$. This high reaction rate provides 


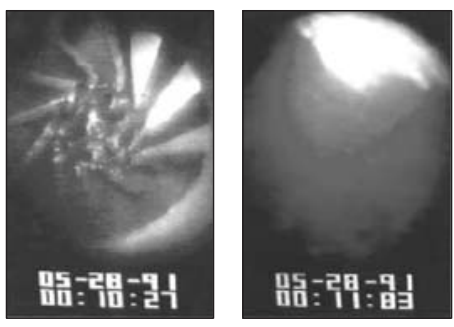

$1.56 \mathrm{~s}$

(a)

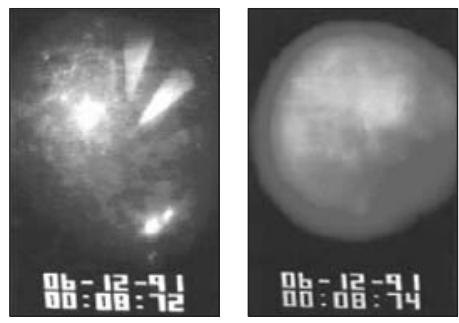

$0.02 \mathrm{~s}$

(b)

Figure 6 Kerosene (a) vs. hydrogen (b) ignition [7]

the first indication of the required response time for the control loop. All the requirements have to be reproducible in order to guarantee the safe operation of the hydrogen-fuelled gas turbine.

\subsection{Determination of the PID Control Parameters}

The target was to find out the combination of PID parameter settings which meet best the defined requirements. Figure 7 shows the simplified graphic presentation of the problem.

The PID control loop was implemented in the control software of the engine. It accords to the following general PID algorithm:

$$
\begin{aligned}
& y(t)=K_{c} e(t)+\frac{K_{c}}{T_{i}} \int_{0}^{t} e(t) d t+K_{c} T_{d} \frac{d e(t)}{d t}+y_{0} \\
& y(k)=K_{c} e(k)+\frac{K_{c} T_{A}}{T_{i}} \sum_{i=0}^{k} e(i)+\frac{K_{c} T_{d}(e(k)-e(k-1))}{T_{A}}+y_{0} ; \\
& y(k)=K_{p} e(k)+K_{i} \sum_{i=0}^{k} e(i)+K_{d}(e(k)-e(k-1)) .
\end{aligned}
$$

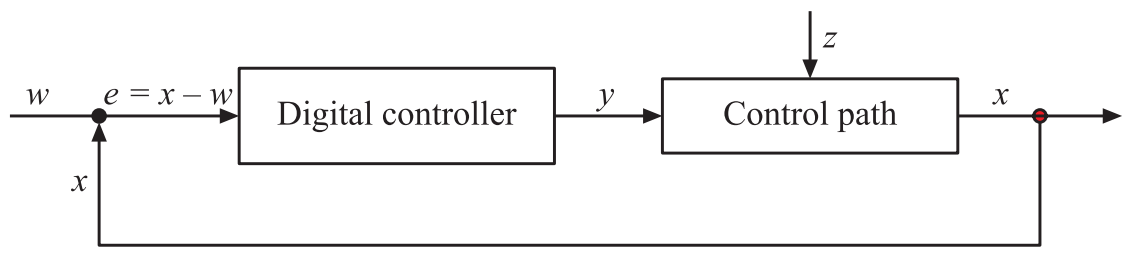

Figure 7 Digital controller 


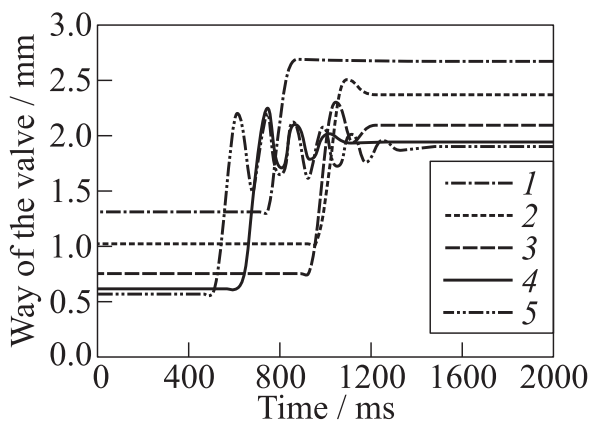

Figure 8 Setting of the $K_{P}$-value: $1-0003 ; 2-0004 ; 3-0006 ; 4-0008$; and $5-0009$

Similar to other industrial applications for determining the PID control parameters for the hydrogen-metering unit control loop, an empirical approach has been chosen in two major steps. First, each single PID parameter was evaluated by giving a control value switch by variegating the parameters of the system. Later on, with the identified PID parameters, a functional test was made in order to validate the settings for the complete operating range of the gas turbine.

For achieving rapidly the target value without oscillating, the first evaluated parameter was the $K_{P}$-element of the control loop. All other elements were switched off. For each tested parameter, the same procedure was passed through. From the same starting basis $(a)$, a control value switch $(2.5 a)$ was given to the system. Figure 8 shows the different step function responses. The values for $P$ elements are given as hexadecimal numeracy.

The values of the $K_{P}$-element were increased up to the point where the system oscillates. With the value from 0009, the system starts to oscillate. According to the Ziegler and Nichols advice for closed loop control [8], the $K_{P}$-value was chosen as $60 \%$ from $K_{P \text { cr }}$ with $K_{P}=0006$. In order to assure that this fact was also adaptive, the following investigations were also done with different $K_{P^{-}}$ values. The attitude of the system was not as satisfying as with the $K_{P}$-value of 0006 .

For avoiding flame blow-off or overheating of the combustion chamber caused by the under- or overshooting of the valve, the $K_{D}$-value had to be adjusted for mimimazing this detrement. Therefore, a value switch was given to the system such that the valve opened from postion $b$ to postion $1.9 b$ (Fig. $9 a$ ). For investigating the system behavior at valve closing, the valve was traversed from $1.9 b$ to $b$ (Fig. $9 b$ ). This procedure was accomplished for different $K_{D^{-}}$ settings. The $K_{I}$-value was still 0000 .

For $K_{D}=0002$, higher overshooting of the valve appeared during valve opening and closing. The attitudes of $K_{D}=0006$ and 0010 were quite similar. 


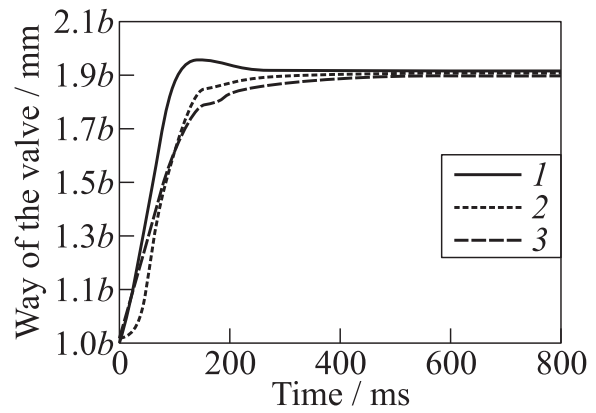

(a)

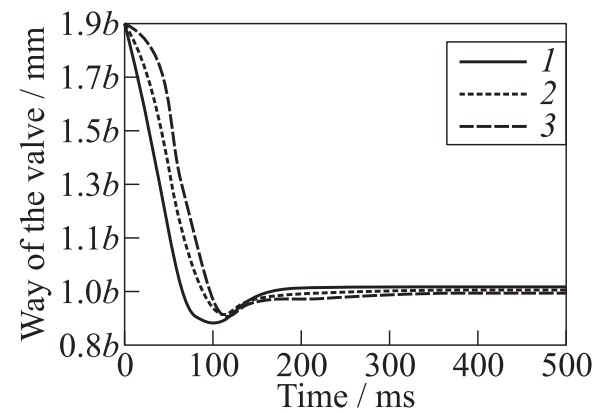

(b)

Figure 9 Setting of the $K_{D}$-value for acceleration $(a)$ and deceleration $(b)$ : 1 $K_{D}=0002 ; 2-0006$; and $3-K_{D}=0010$

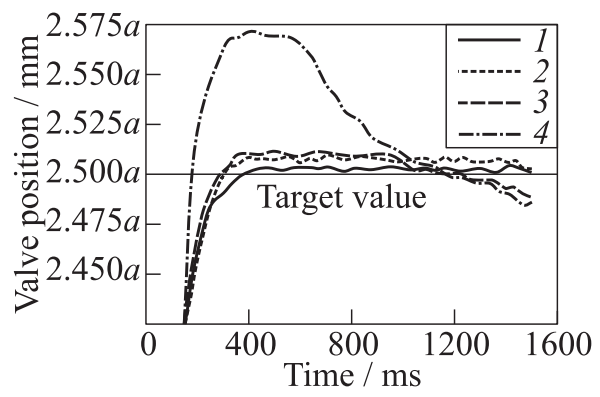

(a)

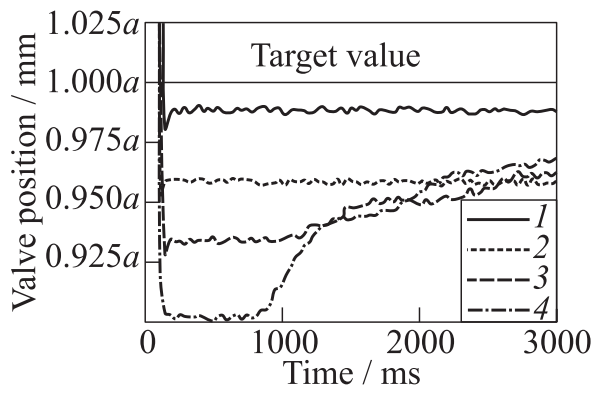

(b)

Figure 10 Setting of the $K_{I}$-value for acceleration $(a)$ and deceleration $(b)$ : 1 $K_{I}=0001 ; 2-0003 ; 3-0006$; and $4-K_{I}=0009$

However, the adjustment of the valve in the $K_{D}=0006$-configuration was a bit faster. For this reason, the value was maintained for this configuration. The spring embedded in the hydraulic cylinder caused the undershooting by the movement from the higher to lower opening positions (see Fig. 9b). The reason for the spring was to secure the closing of the valve when the hydraulic or control system failed. Because the overshooting of the valve was not that high and it was fast corrected by the control loop, it should not have such a great influence on the gas turbine operation.

By setting the $K_{I}$-value, the offset should be reduced and, therefore, a correct metered hydrogen mass flow could be ensured for different operating conditions. The procedure was the same as that for the $K_{D}$-value. Figure 10 presents the system behavior for increasing $K_{I}$-values. For a better outlook, only the final valve positions are presented. With the increase in the $K_{I}$-value, the overshooting of 
the valve also increased. By minimizing, the offset of the $K_{I \text {-value could }}$ not be adjusted to zero. Therefore, the choice of $K_{I}=0001$ was a good agreement.

By setting $K_{P}=0006, K_{D}$ $=0006$, and $K_{I}=0001$, the closed control loop achieved the designated characteristics for engine operation on hydrogen. The valve traversed to the required position rapidly and precisely. In addition, the settled parameters reduced valve by over- and under-

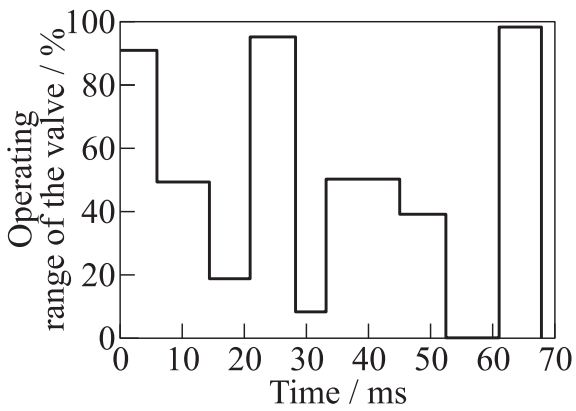

Figure 11 Functionality test shooting. After setting up the PID-element-parameters, the system was tested on functionality. For this reason, the valve was traversed several times for the complete operating range of the gas turbine (Fig. 11).

During the functionality test, the valve always traversed precisely to the required positions and, hence, the accuracy of the metering unit for the engine operation was secured. For opening from zero to the maximum range of the valve, the system needed $0.493 \mathrm{~s}$. During the normal operation of the gas turbine, the valve never did such a step. The new closing of the valve from the maximum position to zero lasted $0.246 \mathrm{~s}$. This value is important for engine emergency shutdown because the fuel supply has to be stopped. It is also important to avoid flame blow-off in the combustion chamber during normal gas turbine operation.

\section{FIRST STARTING TEST AND OUTLOOK}

For testing the starting sequence only the first fuel steps were programmed in the control software. Therefore, after ignition, the engine was accelerated up to $25 \%$ speed. Figure 12 shows one of the first hydrogen starting tests. The ignition of the engine was audible and has been detected.

After acceleration to $7 \%$ speed by the starter, the hydrogen valve was opened and hydrogen was ignited. Thereafter, the temperature in the chamber increased. In comparison to the "starter only" graph, the engine

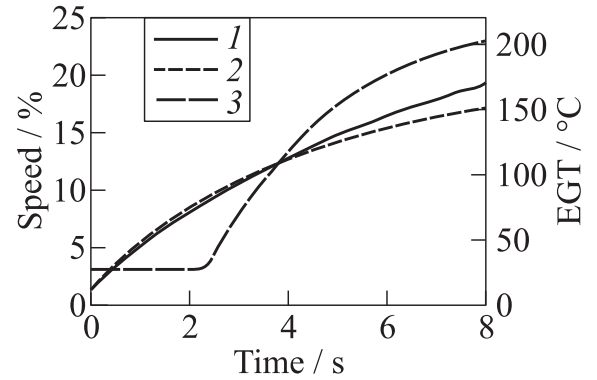

Figure 12 Ignition and acceleration: $1-$ starter + hydrogen; 2 - starter only; and $3-\mathrm{EGT}$ 
was more accelerated by hydrogen burning. To overpower and uncouple the starter, more hydrogen fuel has to be injected in the chamber. As already mentioned, this was the most critical phase during the start sequence because the risk existed to exceed the critical temperatures. Therefore, the approach will be realized in small steps. The setting of PID-elements and therewith the correct operation of the metering unit was an important starting basis for engine operation on hydrogen. The next milestone will be the full acceleration - from ignition up to $95 \%$ speed of the hydrogen engine. Later on, the operating conditions of the engine will be tested.

\section{REFERENCES}

1. Ziemann, J., A. Mayr, A. Anagnostou, F. Suttrop, M. Lowe, S. A. Bagheri, and Th. Nitsche. 1998. Potential use of hydrogen in air propulsion. EQHHPP. Phase III. 0-3. Final Report. Submitted to the European Union.

2. Dahl, G., and F. Suttrop. 2001. CRYOPLANE Project. Combustion Chamber and Emissions, The Micromix Hydrogen Combustor Technology. Task Technical Report 4.4-5A.

3. Suttrop, F., and R. Dorneiski. 1991. Low NOx-potential of hydrogen-fuelled gas turbine engines. 1st Conference (International) on Combustion Technology for Clean Environment. Vilamoura.

4. Suttrop, F. Method and combustor for combusting hydrogen. United States Patent US 6267585 B1, Filed 07.05.1999. Verfahren und Brenner zum Verbrennen von Wasserstoff. German Patent DE 19547506 B4, Filed 19.12.1995. Europen Patent EP 0780631 B1, Filed 10.12.1996.

5. Funke, H.H.-W., A. E. Robinson, and U. Rönna. 2008. Development and testing of a $10 \mathrm{~kW}$ diffusive micromix combustor for hydrogen-fuelled $\mu$-scale gas turbines. ASME Turbo Expo 2008. Berlin. Paper No. GT2008-50418.

6. Recker, E., P. Hendrick, S. Boerner, and H. H.-W Funke. 2009. Flow and application to the "micromix" hydrogen combustion. 19th Symposium (International) on Airbreathing Engines (ISABE). Montreal, Canada.

7. Dahl, G., and F. Suttrop. 1998. Engine control and low-NOx combustion for hydrogen fuelled aircraft gas turbines. Int. J. Hydrogen Energy 23(8):695-704.

8. Ziegler, J. G., and N. B. Nichols. 1942. Optimum settings for automatic controllers. Trans. ASME 64:759-68 